\title{
Uncertain Reasoning in Diagnosis Tools
}

\author{
Xiaofang Deng ${ }^{1}$ Qing Zhou ${ }^{2}$ Gang Lei $^{1}$ \\ ${ }^{1}$ The Software School, Jiangxi Normal University, Nanchang, Jiangxi 330022, P.R.China \\ ${ }^{2}$ The Software Institute, Zhongshan University Guangzhou, Guangdong 510275, P.R.China
}

\begin{abstract}
In this paper we propose a diagnosis system which is based on the knowledge on the systems under diagnoses and the past diagnosis experiences. With the using of uncertain reasoning in the diagnosis system, it can give the most possible solution for the diagnosis system problems which can not be provided by model-based diagnosis systems. A detailed description of the system is given, the whole process of the system is carefully and comprehensively analyzed. Examples in the paper clearly illustrate the advantages of the method in the paper and show how to use the system proposed in the paper.
\end{abstract}

Keywords: Diagnosis, Supporting Degree, Structural Knowledge, Experiences

\section{Introduction}

In this paper we propose a diagnosis system based on knowledge and experiences.

In literature there has been a great deal of research papers in the area published. Many of them utilize the probability theory or fuzzy logic to work on this issue. These approaches, while obviously work in some cases, need experiments or past experiences on diagnosis or a presumption that we could find the probability of the fault of each component. Of course, this restriction is strong in the practical sense, as we might not find such information in many cases. And in these approaches all of our knowledge on the system that we are going to diagnose is not used, but the past experiences and the experiments play the important role. In many cases such experiments say a little on our goal, i.e. these approaches are not always reliable. So some other ways are needed to do the job.

Therefore another approach, model-based diagnosis, was developed. Model-based diagnosis systems formulize the structures of the systems under diagnoses into first order reasoning systems and try to find the faults by using some characters of the reasoning system which contains the knowledge of the structures of the system under diagnosis and the observations of unusual behaviors of components. A typical model for diagnosis was proposed in [6]. This model requires the consistence of $S D \cup D \cup O B S$ for a correct diagnosis $D$, where $S D$ is the set of formulas which contains our knowledge about the system under diagnosis and $O B S$ is the set of formulas containing our observations. So this approach uses the knowledge of the structures of the systems under diagnoses. As our knowledge of the structures of the systems under diagnoses can not be complete in many cases, by the Soundness and Completeness theorem, many formulas in the reasoning system is consistent with $S D \cup D \cup O B S$, so this approach often gives many possible solutions for the diagnosis problem and it has no way to tell which diagnosis is the most possible solution. Another disadvantage of model-based diagnosis system is that the past experiences on the system under diagnosis are completely ignored. In our opinion, such experiences are also valuable knowledge when we want to diagnose a system. Although these experiences are not as solid as the knowledge on the structure of the system under diagnosis, but they do tell us something about what could possibly happen, especially in the situations that the working environment is an important factor for the diagnosis.

It is therefore desirable to have a diagnosis method which has advantages from both diagnosis systems based on probability theory and modelbased diagnosis systems. This means that the system can not only use the knowledge about the structure of the system under diagnosis but also the knowledge of the past diagnosis experiences on that system. The method should give the most possible faults of the system in the reasonable base when we do not have complete knowledge. Also it is obviously required that it can be completely implemented by computers.

So in this paper we propose another diagnosis system $U R D$, which gives the most possible faults of the systems under diagnosis on the base of knowledge of the structure of the system under diagnosis 
and the past experiences of diagnosis of the same system.

If a component is a fault, it will cause unusual behaviors of some components in the system. We first formulize such knowledge $K O$ into a first order reasoning system. Then we use deductive reasoning function in the reasoning system and $O B S$, which contains usual and unusual behaviors of components in the system under diagnosis we have found, to find out which of the components are the possible faults causing such unusual behaviors. Since we use the deduction, some meaningless diagnoses (those which are inconsistent with $K O \cup O B S$ ) will not be included. We can get the same result from this part as what we can have from model-based diagnosis systems. If our knowledge is incomplete, this reasoning function can only give a few diagnoses which are possible faults, as model-based diagnosis systems do. We use HYP to denote the result from this part. Then our next problem is to determine which element in $H Y P$ is the most possible fault.

When we have incomplete knowledge, it is impossible for us to find out the faults definitely. This is an uncertain problem. The best we can expect is to give the components which are most possible to be faulty from our knowledge in the reasonable base. To this end, we employ an uncertain reasoning method proposed in [10]. To determine the most possible faults, the past diagnosis experiences are valuable knowledge from our viewpoints. So we have to formulize our past experiences into the first order logic system $U R D$ like what we have done to the knowledge of the structure of system under diagnosis. Then we use the supporting degree proposed in that paper to find out the most possible faults. So both the knowledge of the system under diagnosis and the past experiences are fully used in determining the possibility of fault of each component. And hence it has quite reasonable foundation.

The paper breaks into three sections. The second section contains the detailed description of $U R D$ and the method of finding the $H Y P$ by using the deductive reasoning function in the $U R D$. The last section mainly deals with the process of finding the most possible faults from $H Y P$. The supporting degrees from angles of both the knowledge on the system under diagnosis and the past diagnosis experiences are clearly defined, which are obviously computer-operatable. With supporting degrees such defined the most possible fault can be easily obtained.

\section{Model for diagnosis}

In this section, we are going to construct our model $U R D$ of diagnosis. This part is a modification of the classical theory of consistency-based diagnosis, which is introduced by [1]-[2], and formalized by [4][6]. The modification is made because we want to use a different reasoning method to make it ready for the processing of determining the most possible faults introduced in the section 3 .

\subsection{The descriptions of the system $U R D$}

The language $L$ of $U R D$ is a first order language, which has:

Variable: which can be assigned the name of a component of the system under diagnosis.

Name: which indicates a component of the system under diagnosis, it is assumed that each component of the system under diagnosis has an exclusive name in $L$.

We use $C O M P$ to denote the finite non-empty set, which contains the names of all components of the system under diagnosis.

Four predicate symbols—Abbe, Abno, Abbe_p, Abno_p; the interpretations of them are:

Abbe: a unary predicate symbol. The formula Abbe $(x)$ represents that the component $x$ behaves abnormally this time. Note that a component behaving abnormally does not mean that it is faulty, because its abnormal behavior may be caused by the abnormal behaviors of other components.

Abno: a unary predicate symbol. The formula $A b n o(x)$ represents that the component $x$ is faulty, and $\neg \operatorname{Abno}(x)$ represents that $x$ is normal.

Abbe_p: a 2-ary predicate symbol. The formula Abbe_p $(x, m)$ represents that the component $x$ was detected to behave abnormally in the $m$-th diagnosis.

Abno_p: a 2-ary predicate symbol. The formula $A b n o \_p(x, m)$ represents that the component $x$ was found to be faulty in the $m$-th diagnosis.

Now we turn to the other parts of $U R D$.

$U R D$ has three inference rules as follows:

If $\alpha$ and $\beta$ are arbitrary formulas, then

$(I R 1)$ infer $\beta$ from $\alpha \longrightarrow \beta$ and $\alpha$

(IR2) infer $\alpha \wedge \beta$ from $\alpha, \beta$

(IR3) infer $\neg \alpha$ from $\alpha \longrightarrow \beta$ and $\neg \beta$

And $U R D$ has four sets of axioms:

${ }^{*} K O$ is a finite set of formulas in $L$, called the system descriptions. Formulas in $K O$ have two types of forms: 
(1) $\operatorname{Abno}(x) \longrightarrow$ Abbe $(x)$;

This formula represents that the fault of the component $x$ causes the abnormal behavior of it. It is assumed that $K O$ has only one such formula for each component of the system.

(2) Abbe $\left(x_{1}\right) \wedge \operatorname{Abbe}\left(x_{2}\right) \wedge \ldots \ldots . \wedge$ Abbe $\left(x_{s}\right) \longrightarrow$ Abbe $(y)$.

This formula represents that all the abnormal behaviors of component $x_{i}$ together $(1 \leq i \leq s)$ cause the abnormal behavior of the component $y$, where $s \geqslant 1$.

We assume that the set $K O$ is consistent.

${ }^{*} O B S$ is called the observations. It's a finite set of closed formulas with the forms Abbe $(a)$ and $\neg$ Abbe $(a)$, which represent the results of our detections, where Abbe (a) represents the behavior of the component $a$ was detected to be abnormal this time, and $\neg A b b e(a)$ represents the behavior of the component $a$ was detected to be normal this time.

We assume that $K O \cup O B S$ is consistent.

${ }^{*} K E$ is a set of formulas which provide links either from the results of current observation to the former detective results of the same component or from current hypothesis to the former diagnosis results about the same component. Formulas in $K E$ have two types of forms:

(1) $\operatorname{Abbe}(a) \longrightarrow$ Abbe_p $(a, m)$

Each of such formulas provides a link from the abnormal behavior of component $a$ to the detective result on $a$ in the $m$ th diagnosis.

(2) $\operatorname{Abno}(a) \longrightarrow$ Abno_p $(a, m)$

Each of such formulas provides a link from our hypothesis to the formula which represents that the component $a$ was diagnosed to be faulty in the $m$ th diagnosis.

${ }^{*} E X P$ is a finite set of formulas in $L$, called the experience set. The set EXP includes one type of formulas: $\quad$ Abbe_p $(y, m) \longrightarrow$ Abno_p $\left(x_{i}, m\right)$, $(1 \leq i \leq k)$.

These formulas come from two facts as follow:

1) The component $y$ was detected to behave abnormally and components $x_{1}, x_{2}, \ldots \ldots ., x_{k}$ were diagnosed to be faulty in the $m$ th diagnosis.

2) The formula $\operatorname{Abbe}\left(x_{1}\right) \wedge \operatorname{Abbe}\left(x_{2}\right) \wedge \ldots \ldots \wedge$ $\operatorname{Abbe}\left(x_{k}\right) \longrightarrow \operatorname{Abbe}(y)$ is consistent with $K O$.

We assume that $K O \cup O B S \cup K E \cup E X P$ is consistent.

In this paper, we assume that all the formulas in $K O$ represent correct relations between components of the system under diagnosis on their abnormal behaviors, but we do not assume that all the relations between components of the system under diagnosis on their abnormal behaviors have been described in $K O$. We also assume that all the formulas in $O B S$ represent correct results of our detections, and all the results of our detections are correct and reliable, but we do not assume that we have detected all the components of the system under diagnosis and all the results are represented in $O B S$. Of course, it is also required that all the formulas in EXP represent the correct information on the past diagnoses. In other words, we assume the correctness of our knowledge, but not the completeness of our knowledge on the system under diagnosis.

\subsection{Discussion on hypothesis}

In our real life a common way of solving diagnosis problem is that: First we assume some components are the faults, and then we try to use our knowledge to prove which of those assumptions are true. To analogue such a process, we give the following definition.

Definition 1 A hypothesis $h$ is a finite non-empty set of closed formulas such that:

i) Every formula in $h$ has the form Abno(a),

ii) if $h=\left\{\operatorname{Abno}\left(a_{1}\right), \operatorname{Abno}\left(a_{2}\right), \ldots \ldots ., \operatorname{Abno}\left(a_{s}\right)\right\}$, then there is a component $b$ in COMP such that the formula Abbe $\left(a_{1}\right) \wedge$ Abbe $\left(a_{2}\right) \wedge \ldots \ldots . \wedge$ Abbe $\left(a_{s}\right) \longrightarrow$ Abbe $(b)$ is included in $K O$, here $s \geq 1$.

The initial hypothesis set INI-HYP is a finite non-empty set, which includes all hypotheses.

From the definition described above, we know that there exists at least one hypothesis for each component of the system under diagnosis, since the formula $A b b e(x) \longrightarrow A b b e(x)$ must be included in $K O$. Then the initial hypothesis set INI-HYP includes a formula $\operatorname{Abno}(x)$ for every component $x$ in $C O M P$.

Now we use an example to illustrate the process that we obtain the initial hypothesis set INI-HYP.

Example 2 Consider Figure.1 which depicts a system with seven switches, referred to as $S_{1}, S_{2}, S_{3}, S_{4}, S_{5}, S_{6}, S_{7}$ and five lights, denoted by $L_{1}, L_{2}, L_{3}, L_{4}, L_{5}$. We need to diagnose the system as in the following figure. 


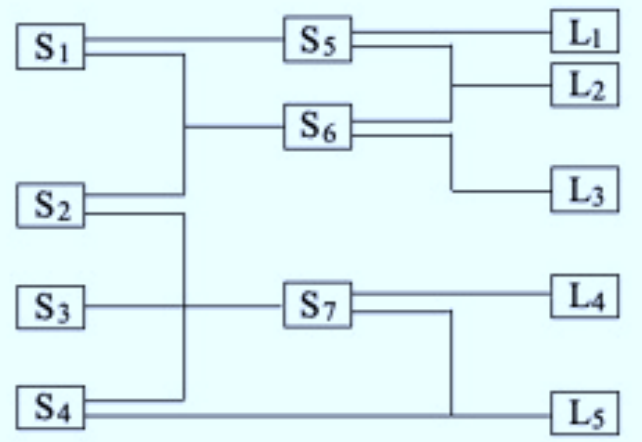

Fig. 1: the Circuit System.

The system description KO is:

$$
\begin{aligned}
& K O=\left\{A b n o(k) \longrightarrow A b b e(k) \mid k \in\left\{S_{1}, S_{2}, S_{3},\right.\right. \\
& \left.\left.S_{4}, S_{5}, S_{6}, S_{7}, L_{1}, L_{2}, L_{3}, L_{4}, L_{5}\right\}\right\} \\
& \cup\left\{\operatorname{Abbe}\left(S_{1}\right) \longrightarrow \operatorname{Abbe}\left(S_{5}\right)\right. \text {, } \\
& \operatorname{Abbe}\left(S_{1}\right) \longrightarrow \operatorname{Abbe}\left(S_{6}\right) \text {, } \\
& \operatorname{Abbe}\left(S_{5}\right) \longrightarrow \operatorname{Abbe}\left(L_{1}\right) \text {, } \\
& \operatorname{Abbe}\left(S_{5}\right) \longrightarrow \operatorname{Abbe}\left(L_{2}\right) \text {, } \\
& \operatorname{Abbe}\left(S_{6}\right) \longrightarrow \operatorname{Abbe}\left(L_{2}\right) \text {, } \\
& \operatorname{Abbe}\left(S_{6}\right) \longrightarrow \operatorname{Abbe}\left(L_{3}\right) \text {, } \\
& \operatorname{Abbe}\left(S_{2}\right) \longrightarrow \operatorname{Abbe}\left(S_{6}\right) \text {, } \\
& \operatorname{Abbe}\left(S_{2}\right) \longrightarrow \operatorname{Abbe}\left(S_{7}\right) \text {, } \\
& \operatorname{Abbe}\left(S_{3}\right) \longrightarrow \operatorname{Abbe}\left(S_{7}\right) \text {, } \\
& \text { Abbe }\left(S_{4}\right) \longrightarrow \operatorname{Abbe}\left(S_{7}\right) \text {, } \\
& \operatorname{Abbe}\left(S_{4}\right) \longrightarrow \operatorname{Abbe}\left(L_{5}\right) \text {, } \\
& \operatorname{Abbe}\left(S_{7}\right) \longrightarrow \operatorname{Abbe}\left(L_{4}\right) \text {, } \\
& \left.\operatorname{Abbe}\left(S_{7}\right) \longrightarrow \operatorname{Abbe}\left(L_{5}\right)\right\}
\end{aligned}
$$

The formula Abno $(k) \longrightarrow$ Abbe $(k)$ represents that the fault of the component $k$ causes the abnormal behavior of it. KO has only one such formula for each component of the system. The formula Abbe $(x) \longrightarrow$ Abbe $(y)$ represents that the abnormal behavior of the component $x$ can cause the abnormal behavior of the component $y$. For example, the formula Abbe $\left(S_{1}\right) \longrightarrow$ Abbe $\left(S_{5}\right)$ represents that the abnormal behavior of $S_{1}$ can cause the abnormal behavior of $S_{5}$.

Now we found that the behavior of the component $L_{4}$ is normal and components $L_{1}, L_{3}$ and $L_{5}$ behave abnormally after detecting the system under diagnosis. Then the observation $O B S$ is:

$O B S=\left\{\operatorname{Abbe}\left(L_{1}\right), \operatorname{Abbe}\left(L_{3}\right), \neg \operatorname{Abbe}\left(L_{4}\right), \operatorname{Abbe}\left(L_{5}\right)\right\}$

We assume that all components in COMP are faulty first, then we have following hypotheses:

$$
h_{1}=\left\{\operatorname{Abno}\left(S_{1}\right)\right\}, \quad h_{2}=\left\{\operatorname{Abno}\left(S_{2}\right)\right\},
$$

$h_{3}=\left\{\operatorname{Abno}\left(S_{3}\right)\right\}, \quad h_{4}=\left\{\operatorname{Abno}\left(S_{4}\right)\right\}$,

$h_{5}=\left\{\operatorname{Abno}\left(S_{5}\right)\right\}, \quad h_{6}=\left\{\operatorname{Abno}\left(S_{6}\right)\right\}$,

$h_{7}=\left\{\operatorname{Abno}\left(S_{7}\right)\right\}, \quad h_{8}=\left\{\operatorname{Abno}\left(L_{1}\right)\right\}$,

$h_{9}=\left\{\operatorname{Abno}\left(L_{2}\right)\right\}, \quad h_{10}=\left\{\operatorname{Abno}\left(L_{3}\right)\right\}$,

$h_{11}=\left\{\operatorname{Abno}\left(L_{4}\right)\right\}, \quad h_{12}=\left\{\operatorname{Abno}\left(L_{5}\right)\right\}$.

And then we have the initial hypothesis set: $I N I-H Y P=\left\{h_{1}, h_{2}, h_{3}, h_{4}, h_{5}, h_{6}, h_{7}, h_{8}, h_{9}, h_{10}\right.$, $\left.h_{11}, h_{12}\right\}$.

But, some hypotheses included in $I N I-H Y P$ are inconsistent with $K O \cup O B S$, which are called improper hypotheses. It is obvious that a hypothesis $h$ is inconsistent with $K O \cup O B S$ if and only if there exists a formula $A b b e(y)$ such that $K O \cup\{h\} \vdash A b b e(y)$ and $K O \cup O B S \vdash \neg A b b e(y)$. We should remove such hypotheses from the set $I N I-H Y P$ firstly when we use $U R D$ to diagnose a system. We use following algorithm to do this job.

Algorithm 3 (In this algorithm, RMV is a temporary set, in which all formulas are negative.)

1. let $R M V:=\phi$

2. for each component $x$ in $C O M P$ do

3. if $\neg A b b e(x) \in O B S$ then

4. $\quad R M V:=R M V \cup\{\neg A b b e(x)\}$

5. for each formula $\neg$ Abbe $(y) \in R M V$ do

6. for each $h \in I N I-H Y P$ do

7. if $K O \cup\{h\} \vdash \operatorname{Abbe}(y)$ then

8. $I N I-H Y P:=I N I-H Y P \backslash h$

9. $H Y P:=I N I-H Y P$

10. return $H Y P$

In this algorithm, the hypothesis set $H Y P$ is the set resulting of the initial hypothesis set INI-HYP after the Algorithm 3 is done, then $H Y P \cup K O \cup O B S$ is consistent. And it is easy to know that $K O \cup O B S \cup H Y P \cup\{\neg \operatorname{Abno}(x): x \in$ $C O M P-H Y P\}$ is consistent, and it is the maximal diagnosis which model-based diagnosis system can get.

Example 4 For the case in Example 2, we found that the behavior of the component $L_{4}$ is normal and components $L_{1}, L_{3}$ and $L_{5}$ behave abnormally.

The component $L_{4}$ behaves normally, then the behavior of the component $S_{7}$ must be normal, and then the behaviors of components $S_{2}, S_{3}$ and $S_{4}$ must be normal.

The normal behavior of a component indicates that it is normal. Then components $L_{4}, S_{7}, S_{2}, S_{3}$ and $S_{4}$ are normal when we do not know whether other components are faulty or not.

Now we use the algorithm 3 to remove those improper diagnoses, which are inconsistent with $K O \cup O B S$, from INI-HYP. 
(1) for the component $L_{4}$, because $\neg$ Abbe $\left(L_{4}\right)$ is included in $O B S$, then $R M V=\left\{\neg A b b e\left(L_{4}\right)\right\}$;

(2) for the formula $\neg A b b e\left(L_{4}\right)$ in $R M V$, because $K O \cup\left\{h_{2}\right\} \quad \vdash \quad \operatorname{Abbe}\left(L_{4}\right), K O \cup\left\{h_{3}\right\} \quad \vdash$ Abbe $\left(L_{4}\right), K O \cup\left\{h_{4}\right\} \vdash \operatorname{Abbe}\left(L_{4}\right), K O \cup\left\{h_{7}\right\} \vdash$ Abbe $\left(L_{4}\right)$ and $K O \cup\left\{h_{11}\right\} \vdash A b b e\left(L_{4}\right)$, then hypotheses $h_{2}, h_{3}, h_{4}, h_{7}$ and $h_{11}$ are removed from the initial hypothesis set INI-HYP.

So we obtain the hypothesis set:

$H Y P=\left\{h_{1}, h_{5}, h_{6}, h_{8}, h_{9}, h_{10}, h_{12}\right\}$.

Then all the formulas which inconsistent with $K O \cup O B S$ are removed from the initial hypothesis set INI-HYP and the formulas remained in $H Y P$ are obviously consistent with $K O \cup O B S$.

It is obvious from the example that all the possible faults are in the $H Y P$, but some hypotheses in $H Y P$ may not be correct diagnoses, so our next job is to determine which of them are most possible diagnoses. This is discussed in the coming section.

\section{Determine the most possible diagnoses}

It is not so hard to see that $H Y P$ is the correct diagnosis when the $K O$ and $O B S$ of the system under diagnosis are complete. So the diagnosis problem can be solved effectively in these situations. But, unfortunately, there are many cases in reality that not enough knowledge of the system under diagnosis is available or some components can not be detected. We have to have a way to determine the correctness of a diagnosis when the information of the system is incomplete.

To solve the problem, we employ the uncertain reasoning method proposed in [10] to $U R D$ to do the job.

Consider the system $U R D$ with $K O, O B S, K E$ and $E X P$ as described in last section. We assume that there are $n$ components in $H Y P$ and their names in $L$ are $x_{1}, x_{2}, \ldots x_{n}$. We call a formula $A$ is a literal if $A$ is an atomic formula or $A$ is a negation of an atomic formula. Then we give the following definitions.

Definition 5 Let $h \in H Y P$ and $A$ is a literal, define:

$$
\begin{aligned}
B_{K O, h}= & \{A \mid A \in L, K O \cup O B S \vdash A\} \\
& \cap\{A \mid A \in L, K O \cup\{h\} \vdash A\} .
\end{aligned}
$$

Giving $h \in H Y P$, formulas in $B_{K O, h}$ have the form $A b b e(a)$, and all of them can be inferred from both $K O \cup O B S$ and $K O \cup\{h\}$. In other words, the abnormal behavior of the component $a$ can be, from the knowledge $K O$ of the system under diagnosis, caused both by the faults of $b_{1}, \ldots, b_{k}$ for $A b n o\left(b_{i}\right) \in$ $H Y P$ and components which are found from $O B S$ that behave abnormally. Then we have reason to believe that all the formulas in $B_{K O, h}$ support $h$, and hence the larger $B_{K O, h}$ is, the more possible $h$ is true. Therefore the following definition makes sense.

Definition $6 \quad T_{h_{i}}^{1}=\frac{\left|B_{K O, h_{i}}\right|}{\underbrace{u}_{h_{j} \in H Y P} B_{K O, h_{j}} \mid}$, for $h_{i} \in$ $H Y P$, here, $|A|$ is the cardinal of the set $A$.

$T_{h_{i}}^{1}$ is called the first supporting degree of $h_{i}$, it shows how much our knowledge of the system under diagnosis supports $h_{i}$ for every $h_{i} \in H Y P$.

Now we use an example to illustrate the process that $U R D$ computes the first supporting degree for each hypothesis in $H Y P$.

Example 7 For the case in Example 4, we can compute the first supporting degree of each hypothesis $h_{i}$ in HYP now.

From the description in Example 4, it is easy to see from our knowledge on the system that the component $S_{1}$ has the largest possibility to be faulty, since its abnormal behavior can cause the abnormal behaviors of both $L_{1}$ and $L_{3}$. Now let us see what $U R D$ will give.

By the definition, we immediately have:

$B_{K O, h_{1}}=\left\{\operatorname{Abbe}\left(L_{1}\right), \operatorname{Abbe}\left(L_{3}\right)\right\}$,

$B_{K O, h_{5}}=\left\{\operatorname{Abbe}\left(L_{1}\right)\right\}, B_{K O, h_{6}}=\left\{\operatorname{Abbe}\left(L_{3}\right)\right\}$,

$B_{K O, h_{8}}=\left\{\operatorname{Abbe}\left(L_{1}\right)\right\}, B_{K O, h_{9}}=\phi$,

$B_{K O, h_{10}}=\left\{\operatorname{Abbe}\left(L_{3}\right)\right\}$,

$B_{K O, h_{12}}=\left\{\operatorname{Abbe}\left(L_{5}\right)\right\}$.

Then the union of all these $B_{K O, h_{i}}$ is:

$$
\begin{aligned}
\underset{h_{i} \in H Y P}{\cup} B_{K O, h_{i}=} & \left\{\operatorname{Abbe}\left(L_{1}\right), \operatorname{Abbe}\left(L_{3}\right),\right. \\
& \left.\operatorname{Abbe}\left(L_{5}\right)\right\} .
\end{aligned}
$$

And hence:

$$
\begin{aligned}
& T_{h_{1}}^{1}=\frac{2}{3}, T_{h_{5}}^{1}=\frac{1}{3}, T_{h_{6}}^{1}=\frac{1}{3}, \\
& T_{h_{8}}^{1}=\frac{1}{3}, T_{h_{9}}^{1}=0, T_{h_{10}}^{1}=\frac{1}{3}, T_{h_{12}}^{1}=\frac{1}{3} .
\end{aligned}
$$

So $h_{1}$ has the largest first supporting degree, i.e., $S_{1}$ has the largest possibility to be faulty from the knowledge on the system under diagnosis, which coincides with what we believe from human thought.

The first supporting degree gives a reasonable suggestion of which components are possible faults but it does not use information of past diagnosis experiences of the same system. Every expert knows 
that the past experiences are valuable factor for diagnosis, so we have to use such knowledge in our system. We begin with the following definition:

Definition 8 Let $h \in H Y P$ and $A$ is a literal, define:

$$
\begin{aligned}
B_{K E, h}= & \{A \mid A \in L, K E \cup O B S \cup E X P \vdash A\} \\
& \cap\{A \mid A \in L, K E \cup\{h\} \vdash A\} .
\end{aligned}
$$

From the definition each formula in $B_{K E, h}$ has the form Abno_p $(x, m)$ which can be inferred from both $K E \cup O B S \cup E X P$ and $K E \cup\{h\}$. So each formula in $B_{K E, h}$ tells us that the components in $h$ were diagnosed to be faulty in the past when same detective results occurred. And then the more formula in $B_{K E, h}$, the more possible $h$ to be true this time. So the following definition makes sense:

Definition $9 \quad T_{h_{i}}^{2}=\frac{\left|B_{K E, h_{i}}\right|}{\bigcup_{h_{j} \in H Y P} B_{K E, h_{j}} \mid}$, for $h_{i} \in$ $H Y P$.

$T_{h_{i}}^{2}$ is called the second supporting degree of $h_{i}$, which gives the supporting degree of $h_{i}$ among all the hypotheses in $H Y P$ from the angle of past experiences. This use of the past experiences is definitely valuable to determine the most possible faults. The following example shows how to calculate it:

Example 10 Suppose in Example 4, the past diagnosis experiences of the same system are listed as table 1:

\begin{tabular}{|c|c|c|}
\hline the mth diagnosis & faulty & behaved abnormally \\
\hline 1 & $S_{1}, S_{2}$ & $L_{1}, L_{3}$ \\
2 & $S_{2}, S_{5}$ & $L_{1}, L_{5}$ \\
3 & $S_{5}, L_{3}$ & $L_{2}, L_{3}$ \\
4 & $S_{1}, S_{7}$ & $L_{3}, L_{4}$ \\
5 & $S_{3}, S_{5}$ & $L_{1}, L_{5}$ \\
6 & $S_{1}, S_{6}$ & $L_{1}, L_{2}$ \\
7 & $S_{4}, S_{5}$ & $L_{1}, L_{4}$ \\
8 & $L_{5}, S_{5}$ & $L_{2}, L_{5}$ \\
\hline
\end{tabular}

Table 1: Formal Diagnosis Results

From the list above, we can see that the component $S_{5}$ was diagnosed to be faulty more frequently than other components when components $L_{1}, L_{3}$ or $L_{5}$ was detected to behave abnormally, then we have reasons to believe that the component $S_{5}$ has the largest possibility to be faulty this time from the angle of our past experiences. Now we use URD to diagnose this system mainly depending on experience.
The experience set are listed as follow: $E X P=\left\{\right.$ Abbe_p $\left(L_{1}, 1\right) \longrightarrow$ Abno_p $\left(S_{1}, 1\right)$, Abbe_p $\left(L_{1}, 1\right) \longrightarrow$ Abno_p $\left(S_{2}, 1\right)$, Abbe_p $\left(L_{3}, 1\right) \longrightarrow$ Abno_p $\left(S_{1}, 1\right)$, Abbe_p $\left(L_{3}, 1\right) \longrightarrow$ Abno_p $\left(S_{2}, 1\right)$,

Abbe_p $\left(L_{2}, 8\right) \longrightarrow$ Abno_p $\left(L_{5}, 8\right)$ Abbe_p $\left(L_{2}, 8\right) \longrightarrow$ Abno_p $\left(S_{5}, 8\right)$, Abbe_p $\left(L_{5}, 8\right) \longrightarrow$ Abno_p $\left(L_{5}, 8\right)$, Abbe_p $\left(L_{5}, 8\right) \longrightarrow$ Abno_p $\left.\left(S_{5}, 8\right)\right\}$.

By definition, we have:

$$
\begin{aligned}
B_{K E, h_{1}=}= & \left\{\text { Abno_p }\left(S_{1}, 1\right), \text { Abno_p }\left(S_{1}, 4\right),\right. \\
& \text { Abno_p } \left.\left(S_{1}, 6\right)\right\}, \\
B_{K E, h_{5}}= & \left\{\text { Abno_p }\left(S_{5}, 2\right), \text { Abno_p }\left(S_{5}, 3\right),\right. \\
& \text { Abno_p }\left(S_{5}, 5\right), \text { Abno_p }\left(S_{5}, 7\right), \\
& \text { Abno_p } \left.\left(S_{5}, 8\right)\right\}, \\
B_{K E, h_{6}}= & \left\{\text { Abno_p }\left(S_{6}, 6\right)\right\}, \\
B_{K E, h_{8}=}= & \phi, \\
B_{K E, h_{9}=}= & \phi, \\
B_{K E, h_{10}=}= & \text { Abno_p } \left.\left(L_{3}, 3\right)\right\}, \\
B_{K E, h_{12}=}= & \left\{\text { Abno_p }\left(L_{5}, 8\right)\right\} .
\end{aligned}
$$

Then the union of these $B_{K E, h_{i}}$ is:

$$
\begin{aligned}
& \bigcup_{h_{i} \in H Y P} B_{K E, h_{i}}=\left\{\text { Abno_p }_{-}\left(S_{1}, 1\right), \text { Abno_p }\left(S_{1}, 4\right),\right. \\
& \text { Abno_p }\left(S_{1}, 6\right), \text { Abno_p }\left(S_{5}, 2\right) \text {, } \\
& \text { Abno_p }\left(S_{5}, 3\right), \text { Abno_p }\left(S_{5}, 5\right) \text {, } \\
& \text { Abno_p }\left(S_{5}, 7\right), \text { Abno_p }\left(S_{5}, 8\right) \text {, } \\
& \text { Abno_p }\left(S_{6}, 6\right), \text { Abno_p }\left(L_{3}, 3\right) \text {, } \\
& \text { Abno_p } \left.\left(L_{5}, 8\right)\right\} \text {. }
\end{aligned}
$$

So the second supporting degree of each hypothesis $h_{i}$ in $H Y P$ is:

$$
\begin{aligned}
T_{h_{1}}^{2} & =\frac{3}{11}, T_{h_{5}}^{2}=\frac{5}{11}, T_{h_{6}}^{2}=\frac{1}{11}, \\
T_{h_{8}}^{2} & =0, T_{h_{9}}^{2}=0, T_{h_{10}}^{2}=\frac{1}{11}, T_{h_{12}}^{2}=\frac{1}{11} .
\end{aligned}
$$

And hence $h_{5}$ has the highest second supporting degree from the calculation of it. This is what exactly we should believe from our past experience.

Now we have supporting degrees from angles of both the structural knowledge of the system under diagnosis and the experiential knowledge. We want to get the supporting degree which have both of them taken account. Here a fact we have to consider: With the increasing of the use of the system, the second supporting degree $T_{h_{i}}^{2}$ will become 
larger and larger while the first supporting degree $T_{h_{i}}^{1}$ keeps unchanged. So we give two unfixed coefficients $\alpha$ and $\beta$ to have the whole supporting degree for each hypothesis $h$ in $H Y P$. This leads the following:

Definition $11 T_{h_{i}}=\alpha T_{h_{i}}^{1}+\beta T_{h_{i}}^{2}$, for each $h_{i} \in$ $H Y P$.

So $T_{h_{i}}$ is the supporting degree of the hypothesis $h_{i}$ which has the consideration of both structural information and experiential information. We have reasons to believe the larger $T_{h_{i}}$ is, the more likely the hypothesis $h_{i}$ is to be true. Therefore it is reasonable to take $T_{h_{i}}$ as a criteria of evaluating all the hypotheses $h_{i}$ for the purpose of determining the most possible diagnosis. And it is obvious that the most reasonable diagnosis is the hypothesis $h_{i}$ with the largest supporting degree $T_{h_{i}}$. Then the output of $U R D$ is $D G S=h_{i}$.

The following example shows how to find out which components are the most possible faults in the Example 2.

Example 12 Since we have the first supporting degree $T_{h_{i}}^{1}$ in Example 7 and the second supporting degree $T_{h_{i}}^{2}$ in Example 10 for each hypothesis $h_{i}$ in $H Y P, T_{h_{i}}$ is easy to have.

Suppose $\alpha=\beta=1$, then:

$$
\begin{aligned}
& T_{h_{1}}=\frac{31}{33}, T_{h_{5}}=\frac{26}{33}, T_{h_{6}}=\frac{14}{33}, \\
& T_{h_{8}}=\frac{11}{33}, T_{h_{9}}=0, T_{h_{10}}=\frac{14}{33}, T_{h_{12}}=\frac{14}{33} .
\end{aligned}
$$

So the hypothesis $h_{1}$ has the largest supporting degree and it makes us have the conclusion that the component $S_{1}$ has the largest possibility to be faulty. So $D G S=\left\{\operatorname{Abno}\left(S_{1}\right)\right\}$.

With the supporting degree defined in Definition 11 , we can find out components which are the most possible faults. But, sometimes, DGS may not cover all positive formulas in $O B S$. This means that some other faults are missed even if $D G S$ is correct. When this occurs, we should compute the supporting degree again after DGS and the formulas in $O B S$ which covered by $D G S$ are removed from $H Y P$ and $O B S$ respectively. Continuing this process we can find out the most possible $D G S$ in a quite reasonable base. The following algorithm is for this process:

Algorithm 13 (This algorithm is to find out those components which have the largest possibilities to be faulty after improper hypotheses are removed from INI-HYP by using of the Algorithm 3.)

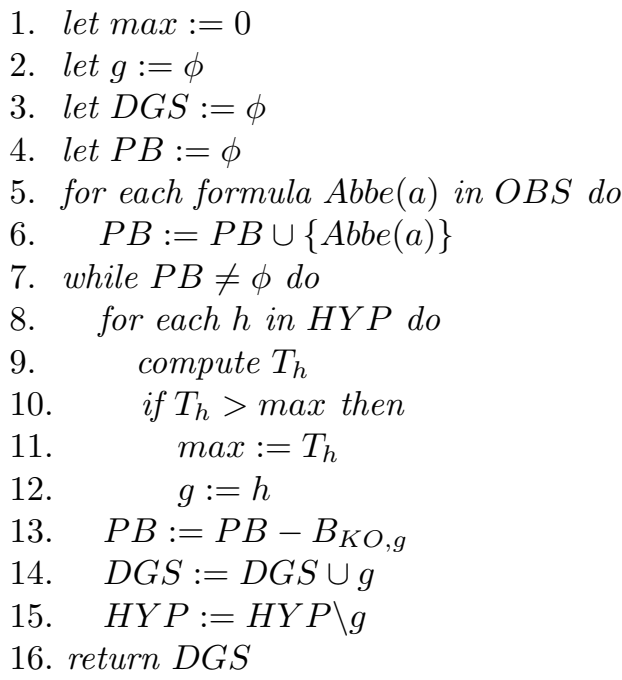

In this algorithm, $P B$ is a temporary set which includes all positive formulas in $O B S$; The set $g$ is a temporary set and $\max$ is a temporary variable.

In step 13, we remove those formulas that support $h$ from the set $P B$. The supporting degree of other hypotheses which are included in $H Y P$ will not be influenced by this removal when we compute their supporting degrees again.

From the algorithm above, it is easy to see that $D G S$ is not only the most possible diagnosis but also the minimal set which is consistent with $K O \cup$ $O B S$. Of course, this result is among diagnoses obtained from model-based diagnosis systems, but they have no way to pick up it from those diagnoses obtained by model-based systems.

The last example is to show how to find $D G S$ by the above algorithm.

Example 14 From the Example 12, DGS $=h_{1}=$ $\left\{\operatorname{Abno}\left(S_{1}\right)\right\}$, but $A b n o\left(S_{1}\right)$ can not cover all positive formulas in $O B S$, as Abbe $\left(L_{5}\right)$ in $O B S$ is uncovered. Then we need to use Algorithm 13 to find out other hypotheses which have the largest possibilities to be true until all positive formulas in $O B S$ are covered.

(1) $P B=\left\{\operatorname{Abbe}\left(L_{1}\right), \operatorname{Abbe}\left(L_{3}\right), \operatorname{Abbe}\left(L_{5}\right)\right\}$;

(2) Because $P B \neq \phi$. From the Example 12, $D G S=h_{1}$;

(3) $P B=P B-B_{K O, g}=P B-B_{K O, h_{1}}=$ $\left\{\operatorname{Abbe}\left(L_{5}\right)\right\}$;

(4) $D G S=\left\{\operatorname{Abno}\left(S_{1}\right)\right\}$

(5) $H Y P=\left\{h_{5}, h_{6}, h_{8}, h_{9}, h_{10}, h_{12}\right\}$;

(6) Because $P B \neq \phi$. By comparing the supporting degrees for those hypotheses still in $H Y P$ from the remaining evidence set $P B$ together with $K O$ and $K E$ with the similar method used in Examples 7, 10 and 12, it follows that $g=h_{12}$; 
(7) $P B=P B-B_{K O, g}=P B-B_{K O, h_{12}}=\phi$;

(8) $D G S=\left\{\operatorname{Abno}\left(S_{1}\right), \operatorname{Abno}\left(L_{5}\right)\right\}$;

(9) $H Y P=\left\{h_{5}, h_{6}, h_{8}, h_{9}, h_{10}\right\}$;

(10) because $P B=\phi$, exit the repetition.

Then DGS of our system is $\left\{\operatorname{Abno}\left(S_{1}\right), \operatorname{Abno}\left(L_{5}\right)\right\}$. So we can make a conclusion that components $S_{1}$ and $L_{5}$ have the largest possibilities to be faulty in Example 2.

\section{References}

[1] J. de Kleer, Local Methods for Localizing Faults in Electronic Circuits, MIT AI Memo, vol. 394, Massachusetts Institute of Technology, Cambridge, MA, 1976.

[2] J. de Kleer, B.C. Williams, Diagnosing multiple faults, Artificial Intelligence, 32: 97-130, 1987.

[3] J. de Kleer, A.K. Mackworth, R.Reiter, Characterizing diagnoses and systems, Artificial Intelligence, 52: 197-222, 1992.

[4] J.Kohlas, B. Anrig, R.Haenni, P.A. Monney, Model-based diagnostics and probabilistic assumption-based reasoning, Artificial Intelligence, 104: 71-106, 1998.

[5] P.J.F. Lucas, Bayesian model-based diagnosis, International Journal of Approximate Reasoning, 27: 99-119, 2001.

[6] R.Reiter, A theory of diagnosis from first principles, Artificial Intelligence, 32: 57-95, 1987.

[7] E. Santos, J.D. Young, Probabilistic temporal networks: A unified framework for reasoning with time and uncertainty, International Journal of Approximate Reasoning, 20: 263-291, 1999.

[8] J. Lunze, F. Schiller, An example of fault diagnosis by means of probabilistic logic reasoning, Control Engineering Practice, 7: 271-278, 1999.

[9] Q. Zhou, S. Ju, The Concept of Uncertain Reasoning and its Supporting Degree, in: Proceedings of the ISCA 9th International Conference, 2000.

[10] Q. Zhou, W. Peng, Uncertain Reasoning and Decision Making, in: Proceedings of International Conference on Intelligent Information Processing (IIP2004), Springer, 2004. 